\title{
Effets comparés de l'alcoolisation aiguë au Koutoukou de vin de palme (boisson alcoolique artisanale) et au Pastis 45 (boisson alcoolique industrielle) sur la mémorisation, chez l'homme
}

\author{
Koffi Mathias YAO*, Kobenan Fiéni Jean-Baptiste ADOU, Pékani Antoine CAMARA, \\ Niangoran François BAKOU, Némé Antoine TAKO et Bialli SERI \\ Laboratoire de Neurosciences, UFR Biosciences, Université de Cocody-Abidjan, 22 BP 1676 Abidjan 22, \\ Côte d'Ivoire. \\ *Auteur correspondant, E-mail : yaomathias@gmail.com
}

\section{RESUME}

Afin de comparer, sur la mémorisation d'informations nouvelles, les effets aigus d'une boisson alcoolique artisanale (Koutoukou de vin de palme) à ceux d'un alcool industriel (Pastis 45), des expériences ont été effectuées chez 396 sujets à jeun. Ces sujets sont des consommateurs occasionnels des deux types d'alcool, de sexe masculin dont 50 sujets témoins et 346 sujets tests répartis en deux groupes : 173 ont consommé du Koutoukou de vin de palme et 173 du Pastis 45. Un exercice de mémoire utilisant neuf images simples d'objets familiers a été utilisé pour évaluer les capacités de mémorisation. Nous avons observé qu'une heure après l'alcoolisation, les performances des sujets tests sont en dessous de celles des sujets témoins et les deux groupes de sujets tests ont des performances semblables comme ce fut le cas avant l'alcoolisation. Cependant, trois heures après cette consommation, les performances des sujets tests au Koutoukou de vin de palme se trouvent largement en dessous de celles des sujets tests au Pastis 45. Ainsi, le Koutoukou de vin de palme, de fabrication artisanale, aurait un effet plus prolongé dans le temps, sur les capacités de mémorisation des consommateurs.

(C) 2011 International Formulae Group. All rights reserved.

Mots clés : mémorisation, performances, alcoolisation, Koutoukou, Pastis 45.

\section{INTRODUCTION}

En Côte d'Ivoire, les eaux-de-vie artisanales dénommées Koutoukou ont été produites avec l'introduction de l'alambic depuis le Ghana voisin, à partir de 1940 (Amany, 1990). Cette boisson artisanale est produite à partir du jus de canne à sucre ou d'un mélange eau-sucre-levure ou encore par le vin de palme (YAO, 2009). Cependant, la prolifération des plantations de palmier à huile encouragée par l'Etat (Cheyns et al., 2001) et le vieillissement de ces plantations ont contribué à l'utilisation préférentielle du vin de palme pour la production du Koutoukou. Dans une étude réalisée par Camara et al. (2008), il a été montré qu'en raison de son coût peu élevé, le Koutoukou, faisait partie des boissons alcooliques les plus consommées en Côte d'Ivoire, surtout, après la dévaluation du francs CFA en janvier 1994. Depuis lors, la paupérisation des couches sociales favorise la prolifération de cette liqueur artisanale et sa substitution aux boissons alcooliques industrielles dont le prix de vente ne cesse 
d'augmenter (Yao, 2009). Or, toutes les études chromatographiques en phase gazeuse montrent que cette liqueur artisanale contiendrait en moyenne 19,55 mg/l de méthanol, $47 \mathrm{mg} / \mathrm{l}$ de propanol et de nombreux alcools supérieurs jusque-là non identifiés (Camara et al., 2004). Aussi, les études d'Amany (1990), de Camara et al. (2008) et de Yao (2009) montrent que plus de $60 \%$ des alcooliques en cours de sevrage, pensionnaires du centre d'accueil de la Croix Bleue ivoirienne sont des consommateurs exclusifs de Koutoukou.

Par ailleurs, il a été montré que, pour une alcoolémie de 0,50 gramme par litre de sang, des troubles capables d'altérer l'exécution de tâches cognitives étaient observés (West et al., 1993). L'alcool, consommé à dose aiguë, entraîne des troubles de la vigilance pouvant aller jusqu'au coma (Anonyme, 2001). Ces troubles portent essentiellement sur la mémoire à court terme, sur les capacités d'abstraction et sur des fonctions plus élaborées telles que la stratégie d'organisation des tâches (Giancola et al., 1996).

Pour toutes ces raisons, nous nous sommes proposé de comparer, sur la vigilance, les effets aigus du Koutoukou de vin de palme à ceux d'un alcool industriel (Pastis 45). Un test de mémoire pouvant évaluer les capacités de mémorisation d'informations nouvelles, a servi d'outil d'évaluation de la vigilance. Pour cela, nous avons opté pour le test de mémoire spatiale utilisant des images simples d'objets familiers. Il s'agit d'étudier l'évocation d'images au cours d'une épreuve de mémorisation d'une disposition spatiale (Adou, 1999).

\section{MATERIEL ET METHODES}

\section{Sujets}

Les expériences ont été effectuées chez 396 sujets, non alcoolo-dépendants, à jeun (depuis au moins 10 heures pour la nourriture et d'au moins 48 heures pour l'alcool), consommateurs occasionnels des deux types d'alcool, de sexe masculin, au Laboratoire de Neurosciences de l'UFR Biosciences (Université de Cocody-Abidjan). L'âge des sujets varie entre 20 ans et 65 ans avec une moyenne de 32 ans. Ces sujets se répartissent comme suit :

- 50 sujets témoins dont 25 ont consommé une solution de Koutoukou de vin de palme à 1 degré $\left({ }^{\circ}\right)$ et 25 une solution de pastis 45 à $1^{\circ}$; - 346 sujets tests dont 173 ont consommé du Koutoukou de vin de palme et 173 du Pastis 45.

\section{Matériel technique}

Le matériel est essentiellement composé d'un alcoomètre centésimal de type GAY LUSSAC, d'une éprouvette graduée de 1000 millilitres $(\mathrm{ml})$, de neuf images d'objets familiers, d'un cadre de neuf cases et d'une mesurette de $125 \mathrm{ml}$.

\section{Solutions alcooliques}

Ces solutions sont celles du Pastis 45 et du Koutoukou de vin de palme. Après avoir procédé à l'évaluation du degré (concentration) alcoolique de chaque boisson au moyen de l'alcoomètre centésimal de GAY LUSSAC qui a indiqué $47^{\circ}$ pour le Koutoukou de vin de palme et $45^{\circ}$ pour le Pastis 45, la préparation des solutions alcooliques s'est faite par des dilutions en vue d'obtenir les concentrations recherchées. Ces dilutions sont opérées en suivant la formule $\mathrm{CiVi}=\mathrm{CfVf}$ où :

Ci représente la concentration initiale ;

Cf est la concentration finale ;

$\mathrm{Vi}$ correspond au volume initial ;

et Vf indique le volume final.

Ainsi, à partir de $900 \mathrm{ml}$ d'une solution-mère de Koutoukou à $47^{\circ}$, contenue dans une éprouvette graduée, l'obtention d'une solution diluée à $45^{\circ}$ s'est faite par ajout de $40 \mathrm{ml}$ d'eau distillée.

De même, une solution de Koutoukou de vin de palme à $1^{\circ}$ et une solution de Pastis 45 à $1^{\circ}$ ont été préparées. Ces deux solutions ont servi de placebo pour les sujets témoins. 


\section{Méthodes}

La masse (poids) du sujet est prise dès son arrivée au Laboratoire. Il est retenu pour les expérimentations si sa masse se situe dans l'intervalle [65-70] kg. Cette restriction permet d'avoir une alcoolémie approximativement identique après la consommation d'une même quantité d'alcool, puisque l'alcoolémie dépend aussi de la masse du sujet. Par ailleurs, l'intervalle choisi [65-70] kg nous permet d'être certain que tous sujets ayant consommé les $125 \mathrm{ml}$ de Pastis 45 ou de Koutoukou de vin de palme à $45^{\circ}$ sont alcoolisés aigus. Cette assurance est donnée, par le calcul de l'alcoolémie selon la formule de Widmark (1932) qui se présente comme suit :

\section{Volume x Pourcentage d'alcool x 0,8 \\ K x Masse de l'individu}

- T étant la valeur de l'alcoolémie ;

- Le volume correspond au volume d'alcool consommé par l'individu en $\mathrm{ml}$;

- $\quad$ La masse de l'individu est en $\mathrm{kg}$;

- $\quad$ K le coefficient de diffusion :

- $\mathrm{K}=0,7$ pour l'homme

- $\mathrm{K}=0,6$ pour la femme.

Pour une quantité de $125 \mathrm{ml}$ d'alcool à $45^{\circ}$, un homme de $65 \mathrm{~kg}$ obtient une alcoolémie de $\mathrm{T}=0,99$ gramme par litre de sang et pour un homme de $70 \mathrm{~kg}$, l'alcoolémie est de $\mathrm{T}=0,92$ gramme par litre de sang.

De même, pour une quantité de $125 \mathrm{ml}$ à $1^{\circ}$, un homme d'une masse de 65 à $70 \mathrm{~kg}$ obtient une alcoolémie d'environ 0,02 gramme par litre de sang.

Après avoir relevé la masse, chaque sujet dispose de cinq minutes pour consommer soit $125 \mathrm{ml}$ de Koutoukou de vin de palme, soit $125 \mathrm{ml}$ de Pastis 45 ; ce qui permet de constituer deux groupes de sujets consommateurs d'alcool. Après la consommation de $125 \mathrm{ml}$ de Koutoukou de vin de palme ou de Pastis 45, chaque sujet doit mémoriser la disposition de neuf images d'objets familiers qui sont: un boubou, un porc, une mangue, une clé, un vélo, un canari, un masque, une machette et un tam-tam. Ces images sont disposées sur neuf cases dans un cadre carré placé devant lui. Le délai entre la présentation des images et le rappel est fixé à quinze secondes. Au cours de cette présentation qui précède l'épreuve (évocation), la disposition des images dans les cases ne change pas (chaque image occupe toujours la même place sur le cadre). Par contre, l'ordre de présentation des images varie d'un essai à l'autre. La disposition de chaque image est montrée successivement pendant cinq secondes au sujet qui reçoit pour consigne de bien retenir la position de l'image dans le cadre. La disposition d'ensemble des neuf images dans les neuf cases n'est pas présentée au sujet; ce qui implique que chaque image soit retirée avant la présentation de la suivante. Concrètement, le sujet doit dire à l'expérimentateur le nom de l'image qui est associé à la case que ce dernier lui présente. L'évocation des neuf cases constitue un essai qui peut être réussi ou pas.

Pour chaque type d'épreuve, le critère de mémorisation de la disposition spatiale est limité à trois essais successifs sans erreur. En cas d'échec, l'épreuve est arrêtée après dix essais. Au cours de l'exercice, les réponses exactes sont confirmées par l'expérimentateur et les erreurs sont signalées au sujet. L'épreuve est effectuée une fois avant la consommation d'alcool pour les sujets tests. Cette épreuve est reprise chaque heure pendant trois heures après l'ingestion de l'alcool.

\section{Analyse statistique}

Pour cette épreuve, l'échantillon est une série d'essais pouvant atteindre le nombre maximal de dix lorsque la mémorisation est tardive ou n'a pas été établie. Le caractère étudié est le nombre d'erreurs par essai. La mémorisation de la position spatiale portant sur neuf images d'objets usuels, le nombre maximal d'erreurs pourra être de neuf. Le 
pourcentage d'erreurs est déterminé de la manière suivante :

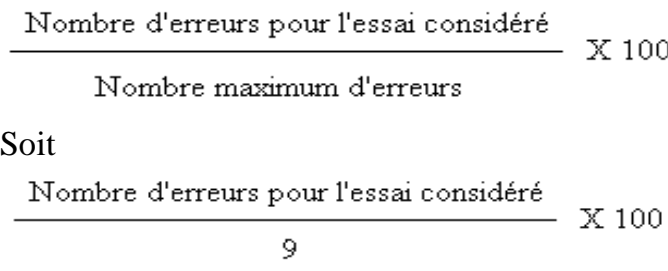

Par ailleurs, à chaque serie d'expériences, trois groupes de sujets sont à comparer: les sujets témoins, les sujets tests au Pastis 45 et les sujets tests au Koutoukou de vin de palme. Il s'agit d'analyser le comportement d'ensemble de chaque groupe à travers leurs performances et de confronter ces résulats aux autres groupes. Ainsi, il convient de vérifier la significativité des différences probables observées entre les moyennes des erreurs obtenues dans chaque groupe, c'est-à-dire savoir si pour chaque épreuve la différence de performances entre deux groupes donnés est significative ou pas. Pour ce faire, une analyse de variance (ANOVA) univariée, grâce au logiciel Statistica 6.0, a permis de faire ces comparaisons, chaque heure.

La probabilité (p) de 0,05 est considérée comme valeur limite de significativité. Ainsi, si «p » est inférieur ou égale à 0,05 , alors la différence entre les variables comparées est significative. Par contre, si «p » est supérieur à 0,05, alors la différence entre les deux variables comparées n'est pas significative.

\section{RESULTATS}

\section{Avant l'alcoolisation}

Avant l'alcoolisation au Pastis 45 et au Koutoukou de vin de palme, tous les sujets soumis à l'épreuve d'évocation (Figure 1), ont réussi leur épreuve avec un maximum de dix essais. Ainsi, les sujets témoins et ceux destinés à la consommation des deux types de boissons alcooliques, ont des performances presque identiques. A ce sujet, la comparaison entre les témoins et les sujets devant consommer du Pastis donne $\mathrm{F}(1,18)=$ 0,00042 et $\mathrm{p}=0,9839$. Cette différence n'est donc pas significative. Aussi, la différence entre les témoins et les sujets destinés à la consommation du Koutoukou de vin de palme n'est également pas significative, car $\mathrm{F}(1,18)$ $=0,0000$ et $p=1$. De même, il n'y a pas de différence significative entre les deux groupes à alcooliser $[\mathrm{F}(1,18)=0,00035 ; \mathrm{p}=0,9852]$.

\section{Une heure après l'alcoolisation.}

A l'épreuve d'évocation, une heure après l'alcoolisation au Pastis 45 et au Koutoukou de vin de palme, la Figure 2 montre que les performances des deux groupes tests diffèrent significativement de celle des témoins.

En effet, entre les sujets consommateurs de Pastis et les sujets témoins, la différence de performances est très significative $[\mathrm{F}(1,18)=18,199$ et $\mathrm{p}=$ 0,00046]. De même, cette différence entre les consommateurs de Koutoukou et les témoins, est également très significative $[\mathrm{F}(1,18)=$ $19,412$ et $\mathrm{p}=0,00034]$.

Les sujets témoins réussissent leur épreuve d'évocation avec un maximum de huit essais, tandis que les deux groupes de sujets tests ne réussissent pas cette épreuve. Aussi, la différence de performances entre ces deux groupes n'est pas significative dans la mesure où $\mathrm{F}(1,18)=0,00074$ pour $\mathrm{p}=$ 0,9785 .

\section{Deux heures après l'alcoolisation}

Deux heures après l'alcoolisation au Pastis 45 et au Koutoukou de vin de palme, à l'épreuve d'évocation, la Figure 3 montre que les performances des deux groupes tests diffèrent de celle des témoins.

En effet, entre les sujets consommateurs de Pastis 45 et les sujets témoins, la différence de performances n'est pas significative $[\mathrm{F}(1,18)=1,4964$ et $\mathrm{p}=$ 0,2370]. Par contre, la différence de performances entre les consommateurs de Koutoukou et les témoins, est significative, car $\mathrm{F}(1,18)=4,9065$ et $\mathrm{p}=0,0399$.

Il convient de signaler que les témoins réussissent leur épreuve d'évocation avec un 
maximum de six essais tandis que les sujets tests au Pastis 45 réussissent cette épreuve avec un maximum de sept essais. Par contre, les sujets tests au Koutoukou ne réussissent pas l'épreuve. Toutefois, la différence de performances entre ces deux groupes tests n'est pas significative, dans la mesure où $\mathrm{F}(1$, 18) $=0,51237$ pour $\mathrm{p}=0,4833$.

\section{Trois heures après l'alcoolisation}

A l'épreuve d'évocation, la Figure 4 indique les performances des deux groupes expérimentaux par rapport aux témoins. En effet, entre les sujets consommateurs de Pastis 45 et les sujets témoins, la différence de performances n'est pas significative $[\mathrm{F}(1,18)$ $=0,07778$ et $\mathrm{p}=0,7835]$. Par contre, la différence de performances entre les consommateurs de Koutoukou de vin de palme et les témoins, est significative $[\mathrm{F}(1$, 18) $=5,9089$ et $p=0,0257]$. De même, la différence de performances entre ces deux groupes tests est également significative, dans la mesure où $\mathrm{F}(1,18)=4,6465$ et $\mathrm{p}=0,0449$.

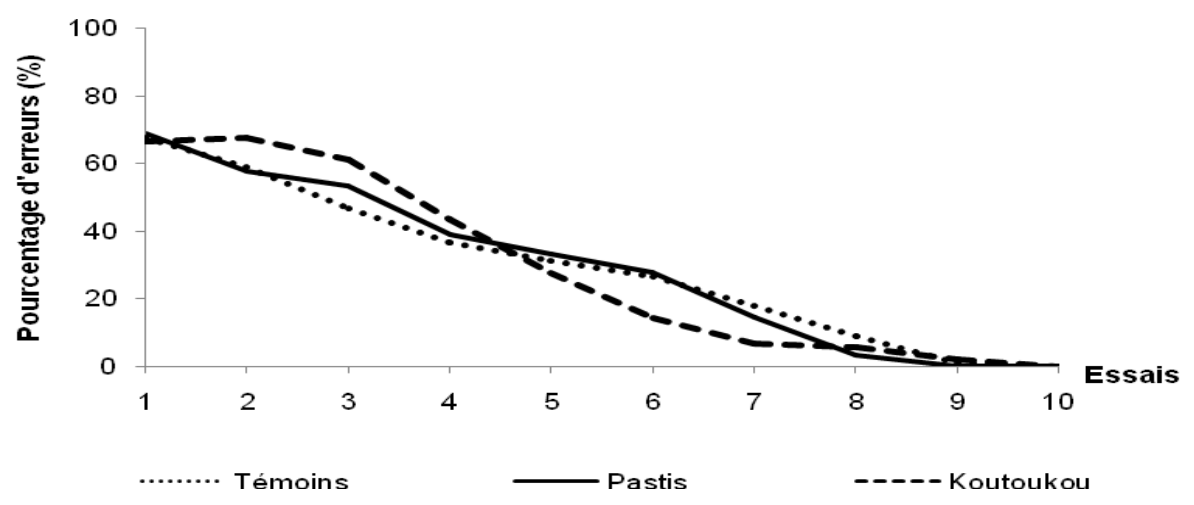

Figure 1 : Performances des sujets à l'épreuve d'évocation, après consommation du placebo et avant la consommation du Pastis 45 et du Koutoukou de vin de palme.

Témoins : groupe de sujets ayant participé au test de mémoire après consommation du placebo. Pastis : groupe de sujets ayant participé au test de mémoire avant la prise du Pastis 45.

Koutoukou : groupe de sujets ayant participé au test de mémoire avant la consommation du Koutoukou de vin de palme. Les performances des trois groupes de sujets sont presque identiques.

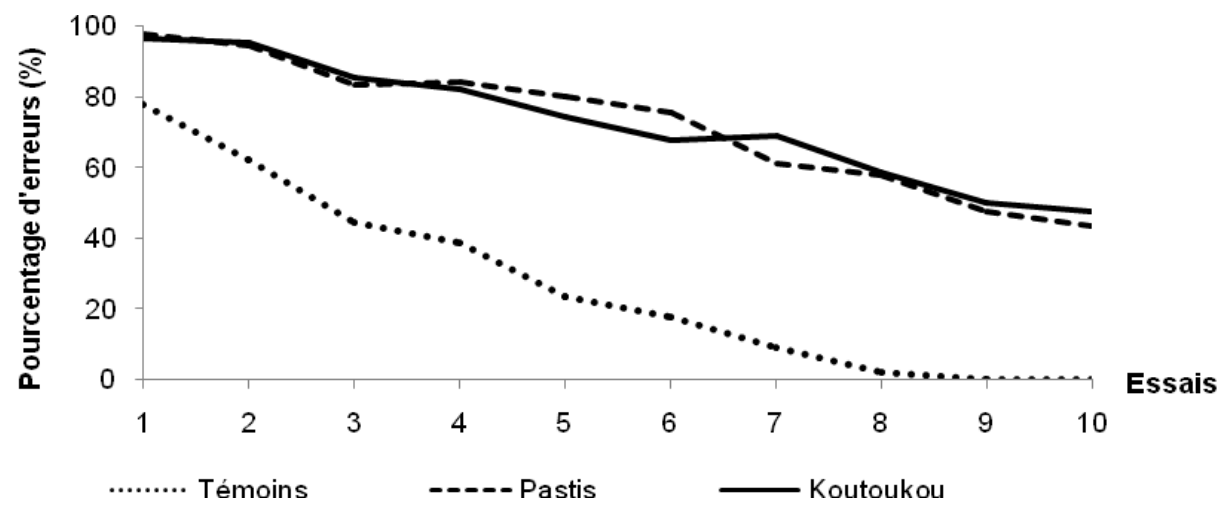

Figure 2 : Performances des sujets à l'épreuve d'évocation, une heure après l'alcoolisation. Témoins : groupe de sujets ayant participé au test de mémoire après consommation du placebo. Pastis : groupe de sujets ayant participé au test de mémoire sous l'influence du Pastis 45.

Koutoukou : groupe de sujets ayant participé au test de mémoire après consommation du Koutoukou de vin de palme. Les sujets sous imprégnation alcoolique au Pastis 45 et au Koutoukou de vin de palme, avec une évolution semblable des performances, ne réussissent pas le test d'évocation. Ils font significativement plus d'erreurs que les sujets témoins. 


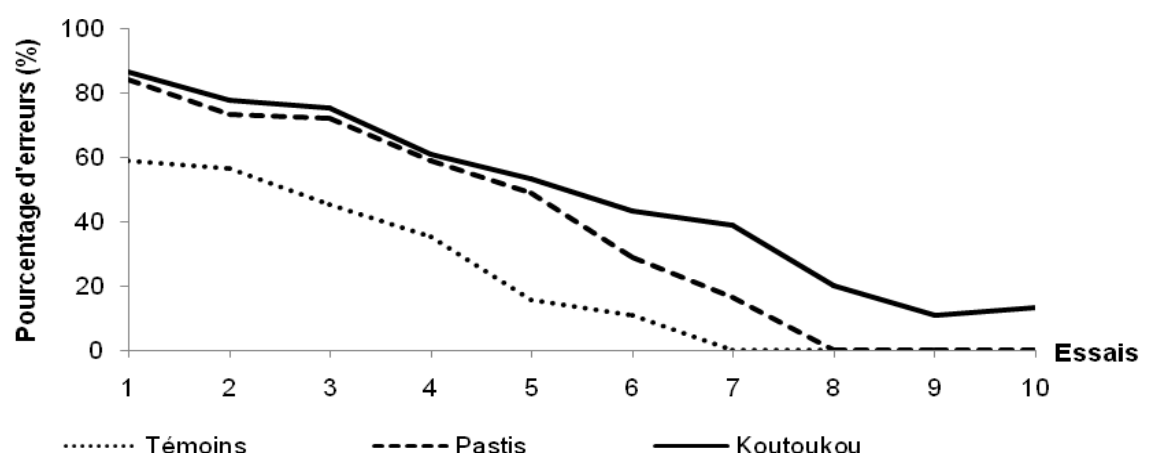

Figure 3 : Performances des sujets à l'épreuve d'évocation, deux heures après l'alcoolisation. Témoins : groupe de sujets ayant participé au test de mémoire après consommation du placebo. Pastis : groupe de sujets ayant participé au test de mémoire sous l'influence du Pastis 45.

Koutoukou : groupe de sujets ayant participé au test de mémoire sous imprégnation au Koutoukou de vin de palme. Les sujets ayant consommé le Koutoukou de vin de palme font plus d'erreurs que les deux autres groupes. Cependant, les sujets témoins restent plus performants que les sujets ayant consommé le Pastis 45.

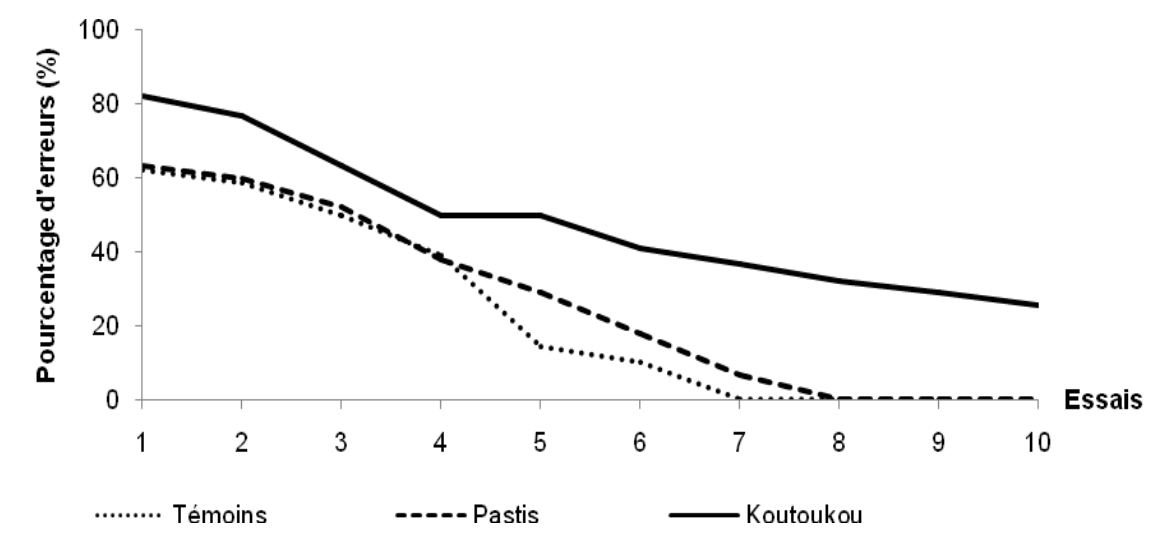

Figure 4 : Performances des sujets à l'épreuve d'évocation, trois heures après l'alcoolisation. Témoins : groupe de sujets ayant participé au test de mémoire après consommation du placebo. Pastis : groupe de sujets ayant participé au test de mémoire sous l'influence du Pastis 45.

Koutoukou : groupe de sujets ayant participé au test de mémoire sous imprégnation au Koutoukou de vin de palme. Les sujets témoins et ceux ayant consommé le Pastis 45 ont presque les mêmes performances alors que les sujets consommateurs de Koutoukou de vin de palme font plus d'erreurs et ne réussissent pas l'épreuve.

\section{DISCUSSION}

Cette étude a porté essentiellement sur des sujets de sexe masculin comme le recommande Ettorre (2004). En effet, la fragilité de la femme face à l'alcool, particulièrement étudiée par Perry (2004), montre que les modifications hormonales engendrées par le cycle menstruel, ne permettent pas de tirer des conclusions efficaces en cas de comparaisons intergroupes.
Par ailleurs, il y avait la nécessité de mettre tous les sujets dans les mêmes conditions psychologiques d'expérience. Cela a donc justifié l'utilisation d'un placebo. S'il est admis pour Oscar-Berman et Marinkovic (2007) que la dose minimale d'alcool pour un effet observable sur le comportement humain serait autour de 0,5 gramme par litre de sang, la présente étude a comparé les performances des sujets témoins ayant consommé le placebo à celles des autres sujets, avant l'ingestion d'alcool par ces derniers. L'absence de 
différence significative des performances qui en est ressortie montre bien, qu'avec une dose d'environ 0,02 gramme par litre de sang pour les sujets témoins, l'alcool n'influence pas significativement leur mémoire. Mais, les 125 $\mathrm{ml}$ d'alcool à $45^{\circ}$ consommés par les sujets tests modifient leur alcoolémie dans un intervalle de [0,92-0,99] gramme par litre de sang. Cette alcoolémie montre que les sujets tests ont ingéré une dose aiguë d'alcool puisque le seuil d'alcoolémie pour atteindre une consommation aiguë est fixée à 0,8 gramme par litre de sang (Anonyme, 2001 ; Parker et al., 2008).

Aussi, une heure après la consommation des deux types d'alcool, une baisse significative des performances des sujets tests s'observe, comparativement aux témoins. Par contre, les deux groupes tests présentent à peu près les mêmes performances. Cela témoignerait d'un trouble transitoire de la mémoire, comme le confirme les travaux de Ben Amar (2007) et ceux d'Apfelbaum et al. (2009). En effet, ces auteurs montrent que l'alcool atteint son maximum de diffusion entre trente minutes et une heure. Cette diffusion rapide concerne principalement les zones les plus irriguées dont le cerveau qui est le siège de la mémoire (De La Torre, 2004 ; Ruitenberg et al., 2005).

Deux heures après la consommation des différentes boissons alcooliques, les performances des deux groupes de sujets tests évoluent différemment. Ce résultat montre, qu'à partir de la deuxième heure après l'alcoolisation, le Koutoukou de vin de palme et le Pastis 45 n'auraient plus les mêmes effets sur la mémoire. Or, après avoir atteint son maximum pendant la première heure, il est évident qu'avec la métabolisation de l'alcool, les effets se réduisent pendant l'heure qui suit. En effet, depuis les travaux de Widmark (1932), l'on estime la valeur de la dégradation de l'éthanol à une vitesse de $0,15 \mathrm{~g} /$ litre/heure. Ainsi, les résultats obtenus avec les sujets tests au Pastis 45 concordent avec les travaux de Koob et Volkow (2010) qui montrent que la dégradation rapide de l'éthanol entrainerait un retour vers une vigilance normale une heure après l'ingestion de l'alcool. Par contre, les effets persistants du Koutoukou pourraient provenir de sa composition en méthanol et en propanol. En effet, l'organisme humain, n'ayant pas d'enzyme spécifique pour métaboliser les autres alcools tels que le méthanol et le propanol, leur dégradation se ferait grâce au même alcool-déshydrogénase, mais de façon plus lente que celle de l'éthanol (Anonyme 2009a, 2009b).

A la troisième heure après l'alcoolisation, on note une différence significative entre les performances des deux groupes tests. Ainsi, les sujets tests au Pastis 45 réussissent l'épreuve d'évocation alors que ceux au Koutoukou de vin de palme ne la réussissent pas. Ce résultat est en accord avec Harris et al. (2003) et Kamarajan et al. (2004) qui indiquent que trois heures après l'ingestion d'une dose aiguë d'éthanol, on observe un retour à la normale de l'activité électroencéphalographique (EEG) spontanée, signe d'un état de vigilance normal. Par ailleurs, les effets du Koutoukou qui perdurent, sont en accord avec les travaux de Camara (1998) qui montrent que quatre heures après la consommation de $125 \mathrm{ml}$ de Koutoukou de vin de palme, les sujets présentent encore des anomalies électroencéphalographiques. Cette observation confirme l'hypothèse selon laquelle le Koutoukou de vin de palme contiendrait d'autres molécules capables de potentialiser les effets de l'éthanol (Hamon et al., 2002) par compétition sur l'alcool déshydrogénase, lors du métabolisme. Ainsi, le méthanol, le propanol et les autres éléments chimiques contenus dans le Koutoukou de vin de palme seraient la cause de la baisse significative des performances des sujets l'ayant consommé. Comme l'ont mentionné Camara et al. (2004), de tels composés chimiques ont des effets très prononcés sur les niveaux de vigilance qui sont en relation avec les niveaux d'activation du système nerveux central d'une part et les niveaux de performances d'autre part. 


\section{Conclusion}

Les troubles cognitifs constituent une complication fréquente résultant de l'usage de l'alcool. En effet, l'état de vigilance des sujets sous imprégnation alcoolique, se trouve effondré. Cependant, si avec la métabolisation, il apparaît certainement une récupération des fonctions cognitives, un retard s'observe avec les sujets tests au Koutoukou de vin de palme. Ainsi, ces derniers font plus d'erreurs à la troisième heure de l'alcoolisation.

Les capacités de mémorisation du sujet, qui dépendent elles-mêmes de facteurs non contrôlables à souhait, constituent alors un moyen efficace pour montrer quelques effets de l'alcoolisation sur la vigilance chez l'humain. Le Koutoukou de vin de palme, de par sa composition chimique particulière, due certainement à son mode de production artisanale, aurait un effet plus prolongé sur les capacités de mémorisation des consommateurs.

\section{REFERENCES}

Adou KFJ-B. 1999. Contribution à l'étude de la trypanosomiase humaine (THA) africaine ou maladie du sommeil, en Côte d'Ivoire (approches électrophysiologique, comportementale et électro-clinique). Thèse de doctorat de 3ème cycle en Psychophysiologie, Université de Cocody-Abidjan, p. 98.

Amany KA. 1990. Etude d'une eau-de-vie traditionnelle : le Koutoukou. Thèse de doctorat de Pharmacie, Université d'Abidjan, p. 118.

Anonyme. 2001. Alcool : effets sur la santé. Expertise collective INSERM (Institut National de la Santé et de la Recherche Médicale). France, 119-273.

Anonyme. 2009a. Méthanol : fiche toxicologique FT 5, INRS (Institut National de Recherche et de Sécurité). France, p.8.

Anonyme. 2009b. Propanol : fiche toxicologique FT 66, INRS (Institut
National de Recherche et de Sécurité). France, p.10.

Apfelbaum M, Romon M, Dubus M. 2009. Ethanol et boissons alcoolisées. In Diététique et Nutrition $\left(7^{\text {th }}\right.$ edn). Elsevier Masson: Paris ; 528.

Ben Amar M. 2007. Les psychotropes criminogènes. Presses Univ. Montréal, 40(1): 11-30.

Camara PA. 1998. Effets de l'intoxication aiguë et chronique au koutoukou (eau-devie traditionnelle africaine) sur le fonctionnement cérébral de l'Homme. Thèse de doctorat d'Etat ès-Sciences (option: Neurosciences et Pharmacopée Africaine), Université d'Abidjan, p. 181.

Camara PA, Yao KM, Adou KFJ-B. 2004. Etude préliminaire des effets d'une prise unique de Koutoukou sur le niveau de vigilance et émotionnel de l'Homme. Rev Inter. Sci. de la Vie et de la Terre, 4(1): 99-113.

Camara PA, Yao KM, Adou KFJ-B, Bakou NF. 2008. Approche épidémiologique de la consommation des boissons alcooliques en Côte d'Ivoire. Rev. Ivoir. Sci. Technol., 12: 157-171.

Cheyns E, Kouamé YS, Nai NS. 2001. Adoption du matériel végétal et itinéraires techniques en plantations villageoises de palmier à huile. Cas de la région des lagunes, Côte d'Ivoire. $O C L$, 8(5) : 524-528.

De La Torre JC. 2004. Is Alzheimer's disease a neurodegenerative or a vascular disorder ? Data, dogma, and dialectics. Lancet Neurol., 3: 184-190.

Ettorre E. 2004. Revisioning women and drug use: gender sensitivity, embodiment and reducing harm. Int. J. Drug Policy, 15(56): 327-335.

Giancola PR, Martin CS, Tarter RE, Pelham WE, Moss HB. 1996. Executive cognitive functioning and aggressive behavior in preadolescent boys at high risk for substance abuse/dependence. J. Stud. Alcohol, 57: 352-359. 
Hamon J-F, Camara PA, Adou KFJ-B, Yao KM. 2002. Goûts et habitude en matière de consommation d'alcool dans le sud et le centre-nord de la Côte d'Ivoire : enquête sur 3428 sujets. Afr. Bioméd., 7(3): 19-26.

Harris DS, Everhart ET, Mendelson J, Jones RT. 2003.The pharmacology of cocaethylene in humans following cocaine and ethanol administration. Drug Alcohol Depend, 72(2): 169-182.

Kamarajan C, Porjesz B, Jones KA, Choi K, Chorlian DB, Padmanabhapillai A, Rangaswamy M, Stimus AT, Begleiter H. 2004. The role of brain oscillations as functional correlates of cognitive systems: a study of frontal inhibitory control in alcoholism. Int. J. Psychophysiol., 51(2): 155-180.

Koob GF, Volkow ND. 2010. Neurocircuitry of addiction. Neuropsychopharm, 35: 217-238.

Oscar-Berman M, Marinkovic K. 2007. Alcohol: Effects on neurobehavioral functions and the brain. Neuropsychol. Review, 17: 239-257.

Parker LLC, Penton-Voak IS, Attwood AS, Munafo MR. 2008. Effects of Acute Alcohol Consumption on Ratings of Attractiveness of Facial Stimuli:
Evidence of Long-Term Encoding. Alcohol Alcoholism, 43(6): 636-640.

Perry BL. 2004. Premenstrual symptomatology and alcohol consumption. J. Stud. Alcohol, 65(4): 464-468.

Ruitenberg A, Den Heijer T, Bakker SL, Van Swieten JC, Koudstaal PJ, Hofman A, Breteler MM. 2005. Cerebral hypoperfusion and clinical onset of dementia: the Rotterdam Study. Ann. Neurol., 57(6): 789-794.

West R, Wilding J, French D, Kemp R, Irving A. 1993. Effect of low and moderate doses of alcohol on driving hazard perception latency and driving speed. Addiction, 88: 527-532.

Widmark EMP. 1932. Die theorischen Grundladen und die praktische Verwendarbeit der gerichtlichmedizinischen Alkohol Bestimmung. Fortsch Naturw Forshung, 11: 140.

Yao KM. 2009. Approche épidémiologique de la consommation d'alcool en côte d'ivoire et évaluation des effets de l'alcoolisation (aiguë et chronique) au koutoukou (eau-de-vie de vin de palme) sur le fonctionnement cérébral des consommateurs. Thèse de doctorat en Physiologie Animale, Université de Cocody-Abidjan, p.151. 